\title{
Clinical Characteristics and Multi-Model Imaging Analysis of Moyamoya Disease: An Observational Study
}

\section{Bing Qin}

Zhejiang University

Liansheng Gao

Zhejiang University

Weilin Xu

Zhejiang University

Tao Li

Zhejiang University

\section{Umut Ocak}

University of Health Sciences

\section{Gao Chen}

Zhejiang University

lin wang ( $\boldsymbol{\nabla}$ dr_wang@zju.edu.cn )

Zhejiang University https://orcid.org/0000-0002-6449-4930

\section{Research Article}

Keywords: Moyamoya disease, clinical manifestations, multi-model, computerized tomography angiography, digital subtraction angiography

Posted Date: June 9th, 2021

DOl: https://doi.org/10.21203/rs.3.rs-576399/v1

License: (a) (1) This work is licensed under a Creative Commons Attribution 4.0 International License. Read Full License 


\section{Abstract}

The study was to analyze the clinical features and imaging characteristics of moyamoya disease (MMD) and to explore the significance of multi-model imaging in the diagnosis of MMD. A retrospective analysis of the clinical features, imaging characteristics, and treatment of 78 adult MMD patients enrolled from March 2010 to March 2013 was performed. The MMD patients in our series were between 23 and 53 years old, and the male to female ratio was 4:1. The main clinical manifestations were sensory abnormalities, headache, and motor dysfunction, depending on the type of MMD. Intracerebral hemorrhage (ICH) was detected in 54 patients (69.2ه), cerebral infarction in 20 (25.6ه), and ICH together with cerebral infarction in 4 (5.1\%). Using computed tomography angiography (CTA), magnetic resonance angiography (MRA), and digital subtraction angiography (DSA), stenotic or occlusive lesions were observed in the internal carotid artery, anterior cerebral artery, and middle cerebral artery. Aneurysms accompanying MMD were found in 17 of the patients (21.8区). Ultimately, 21 patients underwent multiple burr-hole surgery and 57 underwent bypass surgery with or without temporalis muscle sticking. Fourteen patients underwent aneurysm embolization. The clinical manifestations of MMD vary, though the most common is ICH. DSA is the gold standard method for the diagnosis of MMD, and should be performed as early as possible in clinically suspected cases. CTA and MRA are non-invasive techniques used for MMD patient screening and follow-up.

\section{Introduction}

Moyamoya disease (MMD), also known as abnormal basilar vascular network hyperplasia, is a chronic occlusive cerebrovascular disease characterized by stenosis or occlusion of the distal internal carotid artery (ICA) and anterior and middle cerebral arteries accompanied by compensatory dilation of perforating vessels at the base of the brain $(17,27)$. This disease, first defined by Takeuchi and Shimizu in 1957 (23), was named MMD by Suzuki and Takaku in 1967 (31). MMD is most commonly seen in Japan, South Korea, and other East Asian regions (7), and is known to have a broad range of clinical symptoms. Children with MMD often suffer from recurrent cerebral ischemia, resulting in hemiplegia, speech, sensory, and cognitive impairments, mental retardation, and seizures. In contrast, adults with MMD often experience subarachnoid hemorrhage $(\mathrm{SAH})$ or intraparenchymal hemorrhage, resulting in headache, vomiting, meningeal irritation, and symptoms corresponding to the location of the hemorrhage $(12,16)$. Hence, intracerebral hemorrhage $(\mathrm{ICH})$ is more common in adults with MMD than in children with MMD $(1,18)$. Although genetic, inflammatory, and immune factors have been implicated, the exact etiology and pathogenesis of the disease remain unclear (11). MMD is a rare disorder with unspecific clinical manifestations, so it is often misdiagnosed, particularly in the early stages (26). On the other hand, with the popularization of computed tomography angiography (CTA), magnetic resonance angiography (MRA), and digital subtraction angiography (DSA) in recent years, the detection rate of MMD has gradually increased $(35,37,39)$. Among these techniques, DSA is the gold standard for the diagnosis of MMD. 


\section{Materials And Methods \\ 2.1 Ethical review}

This study was approved by the clinical ethics committee of the Second Affiliated Hospital of Zhejiang University School of Medicine. The patients had been anonymized and informed consent was acquired from each patient.

\subsection{General records}

Seventy-eight patients with MMD who were older than 18 and were treated at the Department of Neurosurgery, Second Affiliated Hospital of Zhejiang University School of Medicine between March 2010 and March 2013 were included in this study. Of these 78 patients, 62 were male and 16 were female, and the male to female ratio was $4: 1$. Mean age of symptom onset was $38.50 \pm 8.46$ years (range, 25-53 years).

The distribution of the patients according to age at disease onset is shown in Fig. 1. All patients were diagnosed with MMD by DSA. Fifty-three of the patients presented with the first onset, while 25 presented with the second. The peak age for MMD in our study was between 31 and 45 years, constituting a total of 49 patients $(62.8 \%)$. In addition, the incidence of MMD showed a downward trend after the age of 45 years. Fifty-nine of the patients had a Glasgow coma score (GCS) of 15. Forty-two of the patients had a history of hypertension, but exhibited well-controlled blood pressure at regular check-ups. Fifty-seven of the patients had been cigarette smokers for 5-10 years. None of the patients had a history of alcoholism.

\subsection{Methods}

\subsubsection{Noninvasive imaging examination}

All patients underwent conventional head CT and CTA, and the images were reconstructed using maximum intensity projection technology. Sixty-three patients underwent computed tomography perfusion (CTP). Parameters such as cerebral blood flow (CBF), cerebral blood volume (CBV), mean transmit time (MTT), and time to peak (TTP) were recorded. All patients underwent conventional head magnetic resonance imaging (MRI) and 42 patients underwent MRA. T1-weighted imaging (T1WI), T2weighted imaging (T2WI), and diffusion-weighted imaging (DWI) were performed. MRA was performed using the three-dimensional time-of-flight (3D-TOF) method, and the images were then reconstructed.

\subsubsection{DSA}

All patients underwent DSA. Following right femoral artery puncture and catheterization using the modified Seldinger technique, selective bilateral ICA and bilateral vertebral artery (VA) angiography was performed. Frontal and lateral images in the arterial, capillary, venous, and sinus phases were acquired and reconstructed. The injection rate of contrast medium was $4-5 \mathrm{ml} / \mathrm{s}$.

\subsection{Statistical analysis}


Categorical data are expressed as percentage, and the paired $\chi 2$ test, $\mathrm{K}$ test, or Youden index test is used to compare two diagnostic tests; continuous data are expressed as the mean \pm SD and analyzed by the ttest. A p value $<0.05$ was considered statistically significant. All statistical analyses were conducted using SPSS 13.0.

\section{Results}

\subsection{Clinical manifestations}

The included MMD patients exhibited a range of clinical manifestations: 35 exhibited transient hemiplegia or muscle weakness; 28 exhibited persistent or paroxysmal headache; 23 exhibited dizziness; 19 exhibited moderate unconsciousness (GCS score of 9-12, <30 minutes); 5 exhibited hypoesthesia; 3 exhibited limb numbness; and 1 exhibited epilepsy. Some of the patients exhibited multiple symptoms. None of the patients exhibited significant intellectual impairment. Eight patients presented with their second onset of MMD symptoms.

\subsection{Imaging manifestations}

\subsubsection{CT and MRI}

According to $\mathrm{CT}, 29$ patients were diagnosed with $\mathrm{ICH}, 15$ of which exhibited bleeding into the ventricles, while 31 were diagnosed with SAH, 10 of which exhibited bleeding into the ventricles. Fourteen patients were diagnosed with cerebral infarction, and four were diagnosed with cerebral hemorrhage together with cerebral infarction. According to MRI, 21 patients were diagnosed with $\mathrm{ICH}, 8$ of which exhibited bleeding into the ventricles, and 24 were diagnosed with $\mathrm{SAH}, 9$ of which exhibited bleeding into the ventricles. Twenty-nine patients were diagnosed with cerebral infarction. When the intraoperative results were considered, simple ICH was observed in 54 patients (69.2区), 23 of which exhibited bleeding into the ventricles, while simple cerebral infarction and $\mathrm{ICH}$ together with cerebral infarction were observed in 20 (25.6区) and 4 (5.1\%), respectively. The most common site of cerebral hemorrhage was a ventricle, while the most common sites of cerebral infarction were the frontal lobe and paraventricular area (Fig. 2).

The sensitivity of MRI in diagnosing cerebral hemorrhage was higher than that of CT $(95.6 \%$ vs. $85 \%$, $\mathrm{p}<0.05)$, while the specificity of MRI in diagnosing cerebral hemorrhage was lower than that of CT $(62.1 \%$ vs. $78.6 \%, p<0.05)$. However, the Youden index, which integrates sensitivity and specificity, showed no statistical difference between MRI and CT $(p>0.05)$ in terms of diagnosing cerebral hemorrhage. On the contrary, the sensitivity of MRI in diagnosing cerebral infarction was lower than that of CT $(62.1 \% \mathrm{vs}$. $78.6 \%, p<0.05)$, while the specificity of MRI in diagnosing cerebral infarction was higher than that of CT $(95.6 \%$ vs. $85 \%, p<0.05)$. Analogously, the Youden index showed no statistical difference between MRI and CT ( $p>0.05)$ in terms of diagnosing cerebral infarction. Other detailed diagnostic indexes are listed in Tables 1 and 2.

\subsubsection{CTA}


According to CTA, all patients showed multiple vascular lesions, and bilateral involvement was noted in 63. Overall, 41 patients were diagnosed with ICA lesions, 33 with ACA lesions, and 36 with MCA lesions, all of which manifested as fine, circuitous, stenotic, or occluded vessels. Moyamoya vessels at the base of the brain were found in 43 patients, accompanied by compensatory dilatation of the ipsilateral, contralateral, or bilateral VA, posterior communicating artery (PcoA), or posterior cerebral artery (PCA). Seventeen patients exhibited aneurysms, fourteen of whom exhibited single aneurysms involving the PCA, anterior communicating artery (AcoA), or middle cerebral artery (MCA). The remaining three patients exhibited mutiple small aneurysms manifesting as bilateral spindle aneurysms of the VA and posterior inferior cerebellar artery or multiple small aneurysms of the M1 segment and perforating branch of the right MCA (Fig. 3).

\subsubsection{DSA}

Stenosis or occlusion of the blood vessels and compensatory blood vessels were demonstrated using DSA. Moyamoya vessels at the base of the brain were identified in 53 patients. Furthermore, 50 patients had ICA lesions, 37 had ACA lesions, and 38 had MCA lesions. The compensatory blood supply pattern was also analyzed under dynamic observation: the unilateral or bilateral VA in 51 patients $(65.4 \%)$, the basilar perforating artery in 16 patients $(20.5 \%)$, and the extracranial carotid artery and occipital artery in 7 patients $(9.0 \%)$. Similarly, single aneurysms were found in 14 patients, while multiple small aneurysms were found in 3 patients. Considering DSA as the gold standard imaging technique, the detailed diagnostic indexes of CTA in diagnosing abnormal vessels in MMD are listed in Table 3. The performance of CTA in diagnosing aneurysms was identical to that of DSA (Fig. 4).

\subsubsection{CTP}

CTP showed that the main trunk of the involved artery was not clearly visible, the distal branches of the artery were unevenly developed, and moyamoya vessels were present at the base of the brain. The CBF and CBV of the patients with cerebral infarction were significantly lower than those of the patients with cerebral hemorrhage $(p<0.05)$. The MTT and TTP of the patients with cerebral infarction were significantly higher than those of the patients with cerebral hemorrhage $(p<0.05$, Table 4$)$.

\subsection{Treatment}

The included patients underwent intracranial pressure control, anti-cerebral vasospasm treatment, hemostasis, and other symptomatic treatment when necessary. Overall, 21 patients underwent multiple burr-hole surgery and 57 underwent bypass surgery with or without temporalis muscle sticking. Of the 17 patients with aneurysms, 14 underwent interventional embolization, while 3 did not undergo aneurysm surgery because they had multiple small aneurysms (Fig. 5).

\section{Discussion}


MMD is a chronic cerebrovascular disease presenting with ischemic and/or hemorrhagic manifestations. The pathogenesis of this disease remains unclear, so the currently available treatments have an unfavorable curative effect (29). Pathological changes in the vascular wall have been implicated in MMD pathogenesis, namely fibrocystic proliferation of the vascular intima, significant smooth muscle cell proliferation, and extracellular matrix deposition without phospholipid deposition in the intima and inner elastic layer. These changes are different to those which occur in atherosclerosis $(20,32)$. In addition, Ezura et al. suggested that the disease was related to immune complex-mediated injury (6). Hoshimaru et al. studied studied superficial temporal artery sections and dura mater samples from MMD patients and found increased basic fibroblast growth factor (bFGF) expression in endothelial cells, smooth muscle cells, and the dura mater (10). Similarly, Takahashi et al. demonstrated significantly increased bFGF levels in the cerebrospinal fluid of MMD patients (33). Consequently, bFGF was suggested to promote the proliferation of vascular endothelial cells and smooth muscle cells, resulting in stenosis and occlusion of the ICA and abnormal proliferation of blood vessels in the base of the brain.

In our series, CT showed lower sensitivity and higher specificity than MRI for diagnosing cerebral hemorrhage. Therefore, MRI has a higher ability to detect cases than $\mathrm{CT}$, and patients will rarely be missed, while CT has a higher ability to exclude non-cases than MRI, and almost no non-patients will be misjudged as patients. In other words, MRI demonstrated a lower 'missed-diagnosis' rate and higher 'misdiagnosis' rate than CT. On the contrary, in patients with cerebral infarction, CT demonstrated a lower 'missed-diagnosis'rate and higher 'misdiagnosis' rate than MRI. However, the Youden index, which reflects the overall ability of diagnostic tests to identify patients and non-patients, showed no statistical differences between CT and MRI, regardless of the type of MMD. In addition, MMD-related cerebral hemorrhage often exhibited bleeding into the ventricles; aneurysms and arteriovenous malformations are almost always associated with $\mathrm{SAH}$; whereas hypertension often caused ICH. Indeed, we observed that most hemorrhages were in or around the ventricles such that $29.5 \%$ of the patients in our series exhibited intraventricular hemorrhage. Similarly, the frontal lobe and paraventricular area were the most common sites of MMD-induced cerebral infarction, and multiple areas of cerebral infarction were frequently observed.

All patients in our series were diagnosed by DSA. DSA can clearly show the degree of stenosis or occlusion of the vessels and abnormal neovascularization at the base of the brain, and can also accurately evaluate the pattern and hemodynamics of the compensatory blood supply. We also observed a pattern between the symptoms and imaging findings of MMD. The patients with hemorrhagic symptoms were generally older than those with ischemic symptoms, and showed abundant neovascularization in the base of the brain and ample anastomosis of intracranial and extracranial vessels. Some of the compensatory vessels were tortuous, dilated, and even cystoid. In contrast, the patients with ischemic symptoms were generally younger, and exhibited relatively sparse neovascularization in the base of the brain and poorer collateral circulation. These patients also exhibited a significantly prolonged cerebral circulation time. Variations in clinical symptoms and imaging findings have been suggested to closely correlate with MMD stage. In the early stage, cerebral infarction may occur if neovascularization is not established in time to increase the blood flow to the ischemic area. The 
most common symptoms at this stage are motor and sensory impairments. Once neovascularization is established, the patient experiences a period of blood flow compensation. However, due to the thinness and poor elasticity of the walls of the proliferated vessels, these vessels gradually dilate, distort, and even form aneurysms in response to the continuous pressure exerted by blood, resulting in eventual rupture and bleeding. The most common symptoms at this stage are headache, motor dysfunction, epilepsy, and even unconsciousness.

Recently, CTA and MRA have gained attention with respect to MMD diagnosis. CTA is a convenient, timesaving, and non-invasive technique with good sensitivity and specificity. Importantly, CTA can be performed quickly, which is particularly valuable in pediatric and emergent MMD cases (30). Additionally, CTA can be used to guide surgical treatment, as it allows the relationship between the abnormal blood vessels or aneurysms and the skull to be visualized. Specifically, 4D CTA showed strong consistency and correlation with DSA with respect to vascular stenosis score, but remains insufficient for evaluating the collateral circulation (34). MRA is nonradiative, and the sensitivity and specificity of 3.0T MRA in diagnosing MMD were reported to be $69.2 \%$ and $93.3 \%$, respectively (5). However, compared to DSA, MRA has a tendency to over-diagnose, and often overestimates the degree of vascular stenosis. On the other hand, during the early stages of vascularization or in cases of mild vascular network abnormalities, MRA may fail to diagnose MMD. In addition, the ability of MRA to display collateral circulation and secondary micro-aneurysms is poor. 3.0T TOF MRA, a non-contrast technique, has been proven to be at least equal to CTA for the assessment of internal-external carotid bypass and superior to CTA in the evaluation of the intracranial segment (2). Nevertheless, neither MRA nor CTA were effective at identifying small perforating vessels or early moyamoya vessels. Therefore, different imaging techniques are suited to different situations in line with their advantages and disadvantages (19).

CTP is a sensitive functional imaging technique which can be used to study the cerebral microcirculation, facilitating the evaluation of cerebral perfusion changes in MMD patients before and after surgery (25). Although CBF has been reported to be more strongly correlated with the patency of the bypass artery (3), both CBF and TTP have been demonstrated to be quite sensitive to the presence of altered brain perfusion early after indirect revascularization (4). In line with a previous study (36), using CTP, we showed that adult patients with ischemic MMD exhibited reduced cerebral perfusion compared to patients with hemorrhagic MMD.

Currently, the incidence of intracranial aneurysms in MMD patients is known to range from 3.4 to $14.8 \%$, which is significantly higher than that in the general population (1-3\%) (38). The complex pathogenesis of aneurysms development in MMD may involve multiple factors including hemodynamic disorders and pathological vessel architecture, both of which are known to increase the risk of aneurysm formation (38). Intracranial aneurysms primarily occur in hemorrhagic MMD, and are associated with a high rate of rupture. The rupture of these aneurysms occurs due to the presence of weak and pathological moyamoya vessels (15). Occlusion of the ICA system may result in hemodynamic changes within the circle of Willis by increasing the blood flow in the basilar artery (BA) and PCA. This leads to the development of turbulence in the posterior circulation, which increases the risk of aneurysm formation and subsequent 
rupture. Several authors have suggested that spontaneous occlusion of the MCA may also lead to the focal moyamoya phenomenon and aneurysmal hemorrhages such as cerebral parenchymal hemorrhage, ventricular hemorrhage, and SAH (28). In decreasing order of frequency, the most common locations of aneurysms in MMD patients are the distal anterior choroidal artery (AchA), distal posterior choroidal artery (PchA), AcoA, and BA (22). MMD involving aneurysms can be divided into two types according to the location of the aneurysms: MMD with aneurysms involving the circle of Willis and MMD with peripheral aneurysms involving the moyamoya vessels, which are often located around the ventricles and close to the distal end of the perforating artery, AchA, and PchA (8).

Bypass is the main surgical method used to treat MMD, and involves surgical reconstruction of the collateral circulation to increase cortical perfusion. In 1967, Yasargil performed the first successful STAMCA anastomosis to treat cerebral ischemia in Switzerland. Since then, STA-MCA bypass has been widely used in the treatment of MMD (9). Indeed, collateral vessels, including an intracerebral vascular anastomosis system, corticopial-meningeal vascular anastomosis system, circle of Willis communication system, dural vascular network, and extracranial vascular network, can spontaneously form in MMD patients. However, due to the presence of the meninges, cerebrospinal fluid, and skull, effective collateral circulation cannot be established. The dural and extracranial vascular networks, which comprise many collateral vessels, are unable to be anastomosed to the surface of the brain. However, this can be solved by indirect bypass procedures including encephalo-duro-arterio-synangiosis (EDAS), encephalo-myosynangiosis (EMS), encephalo-duro-arterio-myo-synangiosis (EDAMS). Multiple burr-hole surgery, which are simple, effective, and particularly suitable for anterior circulation ischemia. It has also been suggested that direct and indirect bypass should be combined (21).

In patients with ischemic MMD, direct, indirect, or combined bypass can alleviate ischemic symptoms such as transient ischemic attack (TIA) and reversible neurological deficit (RIND), improve the intellectual capacity of pediatric patients, and effectively prevent the occurrence of future cerebral ischemia $(24,40$, 41). Direct bypass was previously suggested to prevent rebleeding as it can reduce the number and expansion of abnormal branches and the blood flow in the AchA and PcoA (13). This prevents the formation of micro-aneurysms due to abnormal hemodynamics, which are thought to be precursors of hemorrhage or rebleeding (14). In children, indirect bypass is a more effective treatment as the anastomotic vessels are narrow, vulnerable to restenosis, and cannot tolerate direct bypass. The integrity of the STA should be preserved for as long as possible, so that anastomosis can still be performed if necessary. Direct or combined bypass may be more beneficial in adult MMD patients.

\section{Limitations}

This was a single-center study, which may have affected the reliability of the results. Therefore, largerscale, multi-center studies are warranted. Additionally, this study mainly focused on the multi-model imaging and treatment of MMD. However, we did not evaluate the long-term therapeutic effects or followup results. This was beyond the scope of this study, and further research is therefore required. 


\section{Conclusions}

MMD has various clinical and imaging manifestations. Sudden headache due to cerebral hemorrhage is the most common symptom of adult MMD. CT and MRI are valuable in the detection of intracranial hemorrhage and ischemic lesions. CTA and MRA can effectively detect intracranial vascular lesions, while DSA is critical for the screening, diagnosis, and follow-up of MMD. Bypass is the main method currently used to treat MMD.

\section{Abbreviations}

Moyamoya disease (MMD), intracerebral hemorrhage (ICH), digital subtraction angiography (DSA), computed tomography (CT), computed tomography angiography (CTA), magnetic resonance angiography (MRA), computed tomography perfusion (CTP), Glasgow coma score (GCS), cerebral blood flow (CBF), cerebral blood volume (CBV), mean transmit time (MTT), time to peak (TTP), T1-weighted imaging (T1WI), T2-weighted imaging (T2WI), and diffusion-weighted imaging (DWI), three-dimensional time-of-flight (3D-TOF), magnetic resonance imaging (MRI), internal carotid artery (ICA), anterior cerebral artery (ACA), middle cerebral artery (MCA), posterior cerebral artery (PCA), anterior communicating artery $(A \subset O A)$, posterior communicating artery $(P c O A)$, anterior choroidal artery $(A c h A)$, posterior choroidal artery (PchA), basilar artery (BA), vertebral artery (VA), basic fibroblast growth factor (bFGF), subarachnoid hemorrhage (SAH), superficial temporal artery (STA), encephalo-duro-arterio-myo-synangiosis (EDAMS), encephalo-duro-arterio-synangiosis (EDAS), encephalo-myo-synangiosis (EMS), transient ischemic attack (TIA), reversible neurological deficit (RIND).

\section{Declarations}

Funding: This work was supported by the National Natural Science Foundation of China (No.82001299).

Conflict of interests: The authors have no conflict of interests to declare.

Availability of data and material: Not applicable.

Code availability: Not applicable.

Authors' contributions: This study was designed by Bing Qin and Lin Wang, data collection and analysis were conducted by Liansheng Gao, Weilin Xu and Tao Li, this article was written by Bing Qin, Liansheng Gao and Weilin Xu. Language is revised by Umut Ocak. Lin Wang and Gao Chen participated in discussion development and provided expert guidance.

Ethics approval: This study was approved by the clinical ethics committee of the Second Affiliated Hospital of Zhejiang University School of Medicine.

Consent to participate: Not applicable. 
Consent for publication: Not applicable.

Acknowledgments: May the COVID-19 dissipate soon. Salute all the anti epidemic warriors.

\section{References}

1. Bao XY, Wang QN, Zhang Y, Zhang Q, Li DS, Yang WZ, Zhang ZS, Zong R, Han C, Duan L (2019) Epidemiology of Moyamoya Disease in China: Single-Center, Population-Based Study. World neurosurgery 122:e917-e923

2. Chen Q, Qi R, Cheng X, Zhou C, Luo S, Ni L, Huang W (2014) Assessment of extracranial-intracranial bypass in Moyamoya disease using 3T time-of-flight MR angiography: comparison with CT angiography. VASA. Zeitschrift fur Gefasskrankheiten 43(4):278-283

3. Chen Y, Xu W, Guo X, Shi Z, Sun Z, Gao L, Jin F, Wang J, Chen W, Yang Y (2016) CT perfusion assessment of Moyamoya syndrome before and after direct revascularization (superficial temporal artery to middle cerebral artery bypass). European radiology 26(1):254-261

4. Dai DW, Zhao WY, Zhang YW, Yang ZG, Li Q, Xu B, Ma XL, Tian B, Liu JM (2013) Role of CT perfusion imaging in evaluating the effects of multiple burr hole surgery on adult ischemic Moyamoya disease. Neuroradiology 55(12):1431-1438

5. Deng X, Zhang Z, Zhang Y, Zhang D, Wang R, Ye X, Xu L, Wang B, Wang K, Zhao J (2016) Comparison of 7.0- and 3.0-T MRI and MRA in ischemic-type moyamoya disease: preliminary experience. Journal of neurosurgery 124(6):1716-1725

6. Ezura M, Fujiwara S, Nose M, Yoshimoto T, Kyogoku M (1992) Attempts to induce immune-mediated cerebral arterial injury for an experimental model of moyamoya disease. Child's nervous system: ChNS : official journal of the International Society for Pediatric Neurosurgery 8(5):263-267

7. Fujimura M, Bang OY, Kim JS (2016) Moyamoya Disease Frontiers of neurology neuroscience 40:204-220

8. Furtado SV, Medress ZA, Teo M, Steinberg GK (2019) Pathogenesis of aneurysms on major vessels in moyamoya disease and management outcome. Journal of clinical neuroscience: official journal of the Neurosurgical Society of Australasia 61:219-224

9. Heros RC, Morcos JJ (2000) Cerebrovascular surgery: past, present, and future. Neurosurgery 47(5):1007-1033

10. Hoshimaru M, Takahashi JA, Kikuchi H, Nagata I, Hatanaka M (1991) Possible roles of basic fibroblast growth factor in the pathogenesis of moyamoya disease: an immunohistochemical study. Journal of neurosurgery 75(2):267-270

11. Huang S, Guo ZN, Shi M, Yang Y, Rao M (2017) Etiology and pathogenesis of Moyamoya Disease: An update on disease prevalence. Int J Stroke 12(3):246-253

12. Ismail I, Al-Khafaji K, Mutyala M, Aggarwal S, Al-Khafaji N, Kovacs D, Khosla S, Arora R (2015) 'Smoke in the air': a rare cerebrovascular cause of neurological signs and symptoms in a young adult. Journal of community hospital internal medicine perspectives 5(3):27664 
13. Kawaguchi S, Okuno S, Sakaki T (2000) Effect of direct arterial bypass on the prevention of future stroke in patients with the hemorrhagic variety of moyamoya disease. Journal of neurosurgery 93(3):397-401

14. Kikuta K, Takagi Y, Nozaki K, Okada T, Hashimoto N (2007) Histological analysis of microbleed after surgical resection in a patient with moyamoya disease. Neurologia medico-chirurgica 47(12):564567

15. Kim JH, Kwon TH, Kim JH, Chong K, Yoon W (2018) Intracranial Aneurysms in Adult Moyamoya Disease. World neurosurgery 109:e175-e182

16. Kim JS (2016) Moyamoya Disease: Epidemiology, Clinical Features, and Diagnosis. Journal of stroke 18(1):2-11

17. Kuroda S, Houkin K (2008) Moyamoya disease: current concepts and future perspectives. Lancet Neurol 7(11):1056-1066

18. Lee S, Rivkin MJ, Kirton A, deVeber G, Elbers J (2017) International Pediatric Stroke, S. Moyamoya Disease in Children: Results From the International Pediatric Stroke Study. J Child Neurol 32(11):924-929

19. Li J, Jin M, Sun X, Li J, Liu Y, Xi Y, Wang Q, Zhao W, Huang Y (2019) Imaging of Moyamoya Disease and Moyamoya Syndrome: Current Status. J Comput Assist Tomogr 43(2):257-263

20. Lin R, Xie Z, Zhang J, Xu H, Su H, Tan X, Tian D, Su M (2012) Clinical and immunopathological features of Moyamoya disease. PLoS One 7(4):e36386

21. Liu JJ, Steinberg GK (2017) Direct Versus Indirect Bypass for Moyamoya Disease. Neurosurg Clin North Am 28(3):361-374

22. Ni W, Jiang H, Xu B, Lei Y, Yang H, Su J, Gu Y, Mao Y (2018) Treatment of aneurysms in patients with moyamoya disease: a 10-year single-center experience. Journal of neurosurgery 128(6):1813-1822

23. Oshima H, Katayama $Y$ (2012) Discovery of cerebrovascular moyamoya disease: research during the late 1950s and early 1960s. Child's nervous system: ChNS : official journal of the International Society for Pediatric Neurosurgery 28(4):497-500

24. Park SE, Kim JS, Park EK, Shim KW, Kim DS (2018) Direct versus indirect revascularization in the treatment of moyamoya disease. Journal of neurosurgery 129(2):480-489

25. Sasagawa A, Mikami T, Hirano T, Akiyama Y, Mikuni N (2018) Characteristics of cerebral hemodynamics assessed by CT perfusion in moyamoya disease. Journal of clinical neuroscience: official journal of the Neurosurgical Society of Australasia 47:183-189

26. Sasannejad P, Rezaei F, Bidaki R, Zarepur E (2018) Rare Presentation of Moyamoya Disease with Sub acute Presentation in Iran. Iranian journal of child neurology 12(1):89-93

27. Scott RM, Smith ER (2009) Moyamoya disease and moyamoya syndrome. N Engl J Med $360(12): 1226-1237$

28. Seki Y, Fujita M, Mizutani N, Kimura M, Suzuki Y (2001) Spontaneous middle cerebral artery occlusion leading to moyamoya phenomenon and aneurysm formation on collateral arteries. 
Surgical neurology 55(1):58-62 discussion 62

29. Shang S, Zhou D, Ya J, Li S, Yang Q, Ding Y, Ji X, Meng R (2018) Progress in moyamoya disease. Neurosurgical review

30. Sugino T, Mikami T, Ohtaki S, Hirano T, lihoshi S, Houkin K, Mikuni N (2013) Assessment of moyamoya disease using multidetector row computed tomography. Journal of stroke cerebrovascular diseases: the official journal of National Stroke Association 22(5):644-649

31. Suzuki J, Takaku A (1969) Cerebrovascular "moyamoya" disease. Disease showing abnormal net-like vessels in base of brain. Arch Neurol 20(3):288-299

32. Takagi Y, Hermanto Y, Takahashi JC, Funaki T, Kikuchi T, Mineharu Y, Yoshida K, Miyamoto S (2016) Histopathological Characteristics of Distal Middle Cerebral Artery in Adult and Pediatric Patients with Moyamoya Disease. Neurologia medico-chirurgica 56(6):345-349

33. Takahashi A, Sawamura Y, Houkin K, Kamiyama H, Abe H (1993) The cerebrospinal fluid in patients with moyamoya disease (spontaneous occlusion of the circle of Willis) contains high level of basic fibroblast growth factor. Neurosci Lett 160(2):214-216

34. Tian B, Jiang Y, Kang Q, Xu B, Liu R, Liu Q, Lu J (2018) Comparative study of 4D CTA and DSA for vascular assessment in moyamoya disease. Clinical imaging 48:74-78

35. Tomura N, Kokubun M, Horiuchi $\mathrm{K}$, Watanabe Z Comparison of TOF-MRA and silent scan-MRA in depicting cerebral arteries in patients with Moyamoya disease. Acta radiologica:284185118824782; 2019

36. Xie A, Luo L, Ding Y, Li G (2015) Ischemic and hemorrhagic moyamoya disease in adults: CT findings. Int J Clin Exp Med 8(11):21351-21357

37. Zhang J, Wang J, Geng D, Li Y, Song D, Gu Y (2013) Whole-brain CT perfusion and CT angiography assessment of Moyamoya disease before and after surgical revascularization: preliminary study with 256-slice CT. PLoS One 8(2):e57595

38. Zhang L, Xu K, Zhang Y, Wang X, Yu J (2015) Treatment strategies for aneurysms associated with moyamoya disease. International journal of medical sciences 12(3):234-242

39. Zhao Y, Lu J, Zhang Q, Zhang Y, Zhang D, Wang R, Zhao Y (2018) Time Course of Neoangiogenesis After Indirect Bypass Surgery for Moyamoya Disease: Comparison of Short-term and Long-term Follow-up Angiography. Clinical neuroradiology

40. Zhao Y, Yu S, Lu J, Yu L, Li J, Zhang Y, Zhang D, Wang R, Zhao Y (2018) Direct Bypass Surgery Vs. Combined Bypass Surgery for Hemorrhagic Moyamoya Disease: A Comparison of Angiographic Outcomes. Front Neurol 9:1121

41. Zheng J, Yu LB, Dai KF, Zhang Y, Wang R, Zhang D (2019) Clinical Features, Surgical Treatment, and Long-Term Outcome of a Multicenter Cohort of Pediatric Moyamoya. Front Neurol 10:14

\section{Tables}

Table 1. Comparisons of CT and MRI in diagnosing intracerebral hemorrhage of MMD 


\begin{tabular}{|lllllll|}
\hline Cerebral hemorrhage & Sensitivity (\%) & Specificity (\%) & LR+ & LR- & DOR & Youden index \\
\hline CT & 85.0 & 78.6 & 3.97 & 0.19 & 20.89 & 0.64 \\
\hline MRI & 95.6 & 62.1 & 2.52 & 0.07 & 36.00 & 0.58 \\
\hline p value & 0.039 & 0.039 & - & - & - & 0.68 \\
\hline
\end{tabular}

$\mathrm{CT}$, computed tomography; MRI, magnetic resonance imaging; DOR, diagnostic odds ratio; LR+, positive likelyhood ratio; LR-, negative likelyhood ratio.

Table 2. Comparisons of CT and MRI in diagnosing cerebral infarction of MMD

\begin{tabular}{|lllllll|}
\hline Cerebral infarction & Sensitivity (\%) & Specificity (\%) & LR+ & LR- & DOR & Youden index \\
\hline CT & 78.6 & 85.0 & 5.24 & 0.25 & 20.96 & 0.64 \\
\hline MRI & 62.1 & 95.6 & 14.11 & 0.40 & 35.28 & 0.58 \\
\hline p value & 0.039 & 0.039 & - & - & - & 0.68 \\
\hline
\end{tabular}

$\mathrm{CT}$, computed tomography; MRI, magnetic resonance imaging; DOR, diagnostic odds ratio; LR+, positive likelyhood ratio; LR-, negative likelyhood ratio.

Table 3. The accuracy of CTA in diagnosing lesion vessels of MMD

\begin{tabular}{|lllllllll|}
\hline Vessels & $\begin{array}{l}\text { Sensitivity } \\
(\%)\end{array}$ & $\begin{array}{l}\text { Specificity } \\
(\%)\end{array}$ & LR+ & LR- & DOR & $\begin{array}{l}\text { Youden } \\
\text { index }\end{array}$ & $\begin{array}{l}\mathrm{k} \\
\text { value }\end{array}$ & $\begin{array}{l}\mathrm{p} \\
\text { value }\end{array}$ \\
\hline ICA & 90.2 & 64.9 & 2.57 & 0.15 & 17.13 & 0.55 & 0.558 & $<0.001$ \\
\hline ACA & 78.8 & 75.6 & 3.23 & 0.28 & 11.54 & 0.54 & 0.535 & $<0.001$ \\
\hline MCA & 88.9 & 85.7 & 6.22 & 0.13 & 47.85 & 0.75 & 0.743 & $<0.001$ \\
$\begin{array}{l}\text { Moyamoya } \\
\text { vessels }\end{array}$ & 86.0 & 54.3 & 1.88 & 0.25 & 7.52 & 0.40 & 0.414 & $<0.001$ \\
\hline
\end{tabular}

CTA, computed tomography angiography; DSA, digital subtraction angiography; LR+, positive likelyhood ratio; LR-, negative likelyhood ratio; DOR, diagnostic odds ratio; ICA, internal carotid artery; ACA, anterior cerebral artery; MCA, middle cerebral artery.

Table 4. Comparisons of CTP between hemorrhagic and ischemic MMD 


\begin{tabular}{|lllll|}
\hline & $\mathrm{CBV}(\mathrm{ml} / 100 \mathrm{~g})$ & $\mathrm{CBF}(\mathrm{ml} / 100 \mathrm{~g} \cdot \mathrm{min})$ & $\mathrm{MTT}(\mathrm{s})$ & $\mathrm{TTP}(\mathrm{s})$ \\
\hline Cerebral hemorrhage & $2.21 \pm 0.37$ & $33.78 \pm 4.22$ & $5.89 \pm 0.12$ & $12.27 \pm 2.19$ \\
\hline Cerebral infarction & $1.09 \pm 0.31$ & $16.23 \pm 2.87$ & $8.96 \pm 0.77$ & $16.29 \pm 4.12$ \\
\hline p value & 0.012 & 0.016 & 0.026 & 0.043 \\
\hline
\end{tabular}

CTP, computed tomography perfusion; $\mathrm{CBV}$, cerebral blood volume; CBF, cerebral blood flow; MTT, mean transmit time; TTP, time to peak.

\section{Figures}

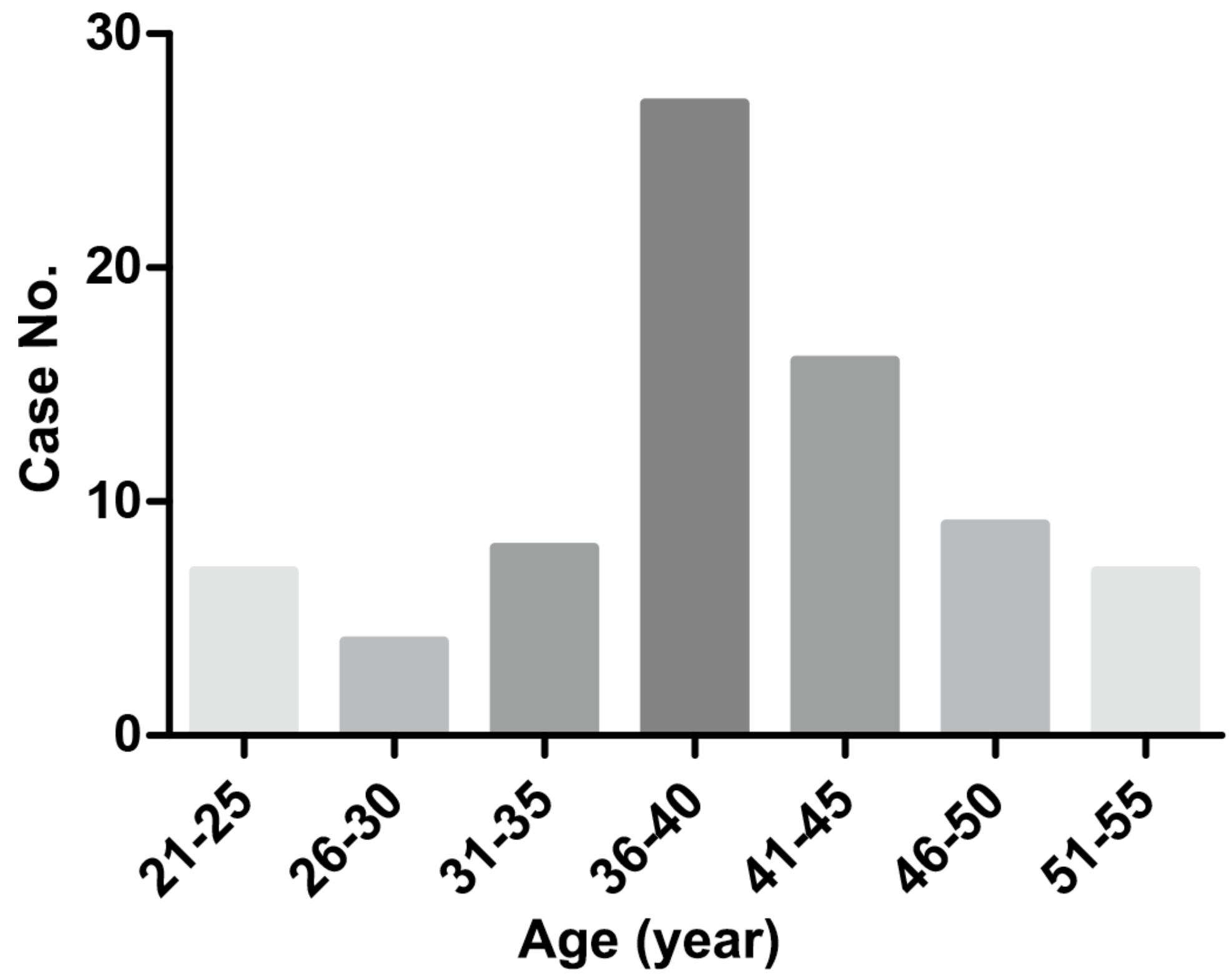

Figure 1

The age distribution of MMD patients. 

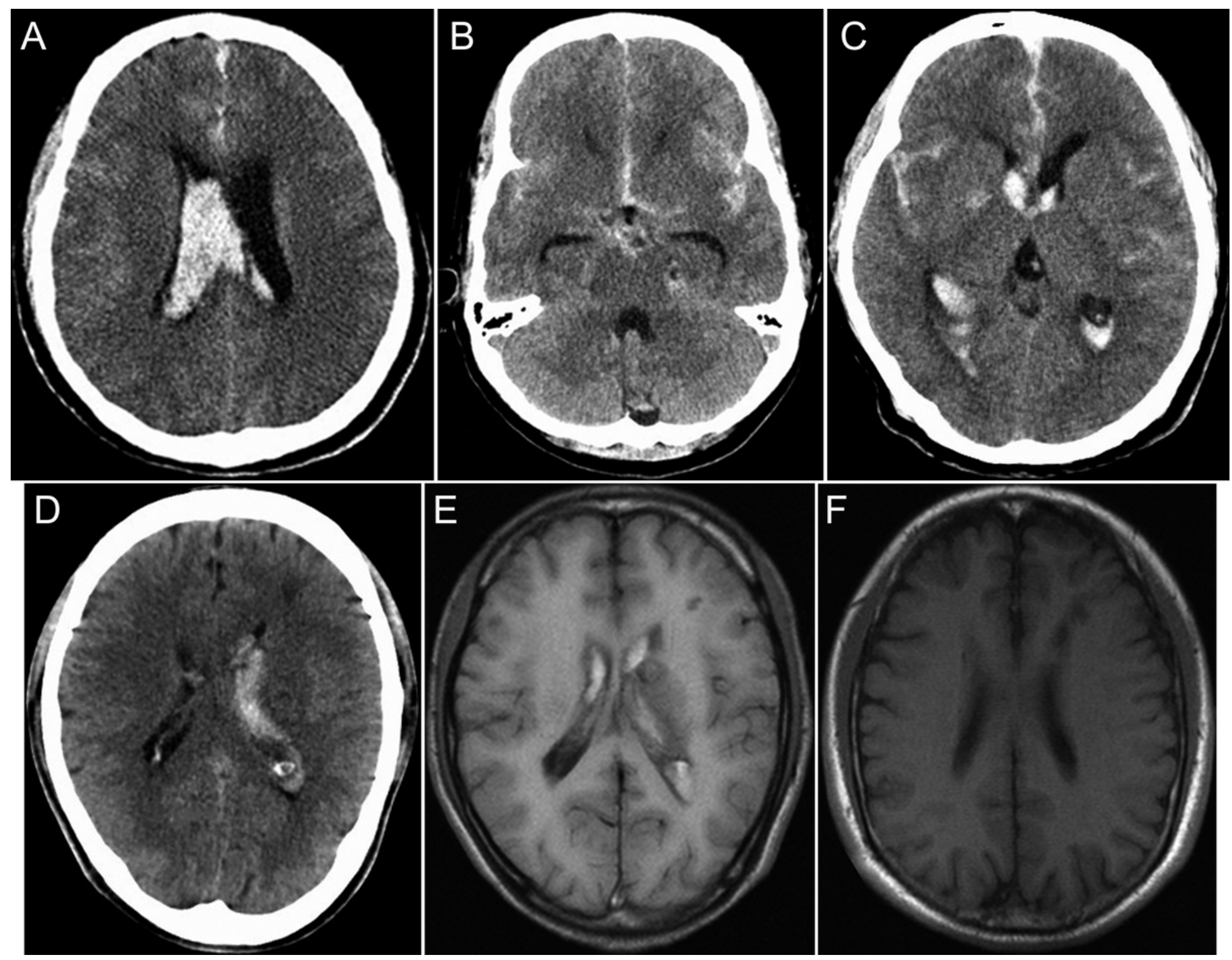

Figure 2

Common imaging signs of MMD. (A) Intraventricular hemorrhage (CT); (B) subarachnoid hemorrhage (CT); (C) subarachnoid hemorrhage breaking into ventricles (CT); (D, E) intraventricular hemorrhage with left frontal lobe infarction (CT and MRI T1WI); (F) frontal lobe infarction (MRI T1WI).
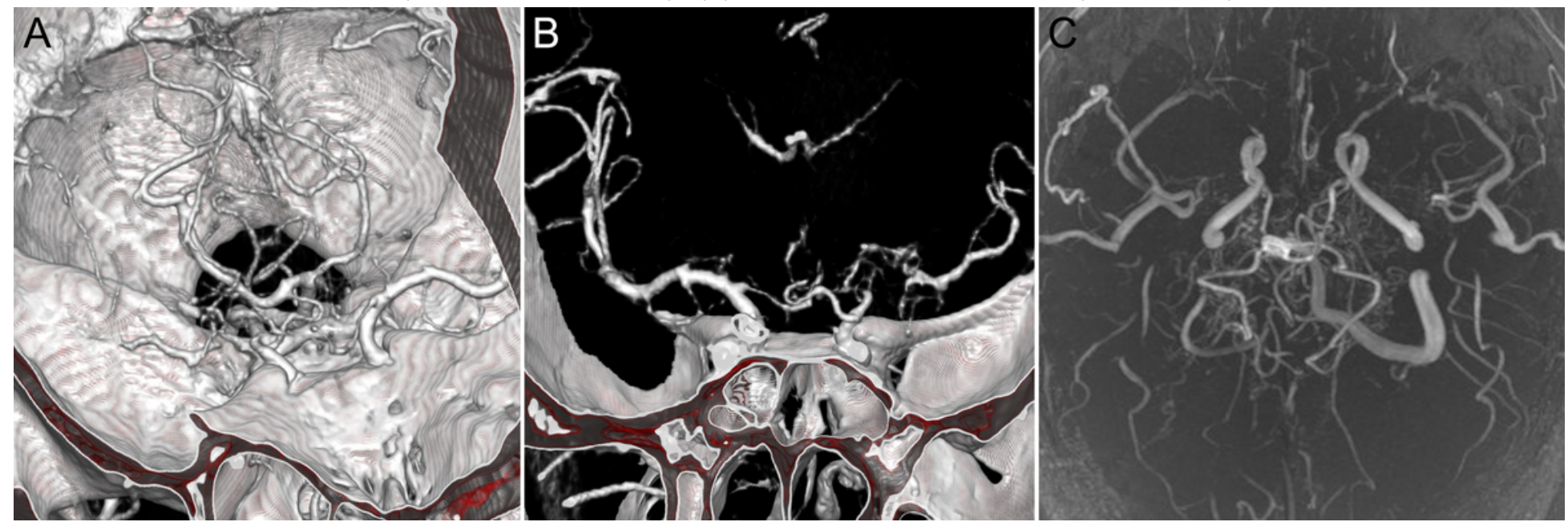


\section{Figure 3}

CTA and MRA manifestations of MMD. (A, B) Network of abnormally dilated collateral vessels at the base of the brain (puff of smoke sign) (CTA); (D) bilateral ICA occlusion and smoky vessels at the base of the brain (MRA).

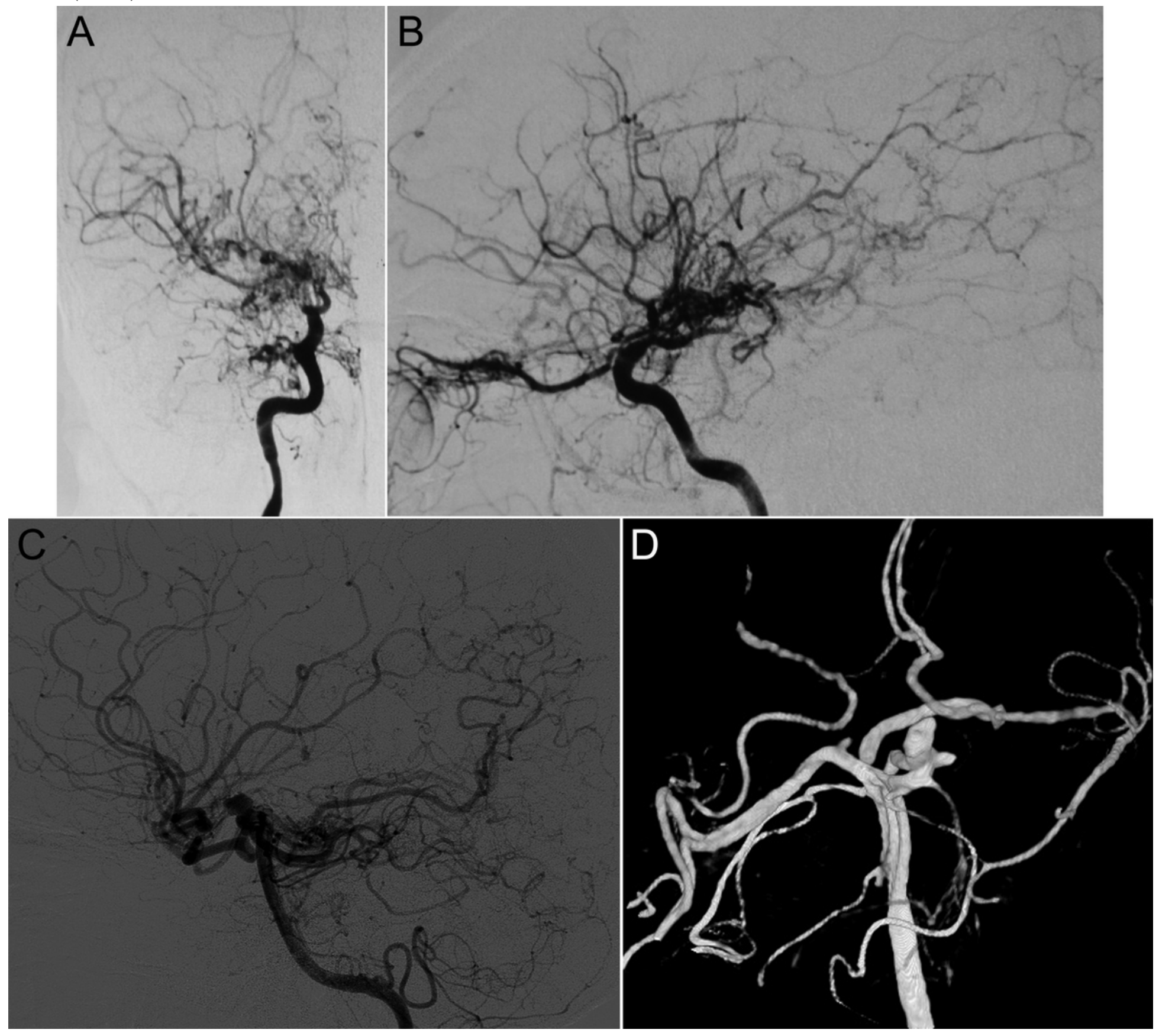

\section{Figure 4}

DSA manifestations of MMD. (A, B) ICA occlusion and smoky vessels at the base of the brain (frontal and lateral views, DSA); (C, D) MMD with PCA aneurysm (lateral view of DSA and 3D reconstruction). 

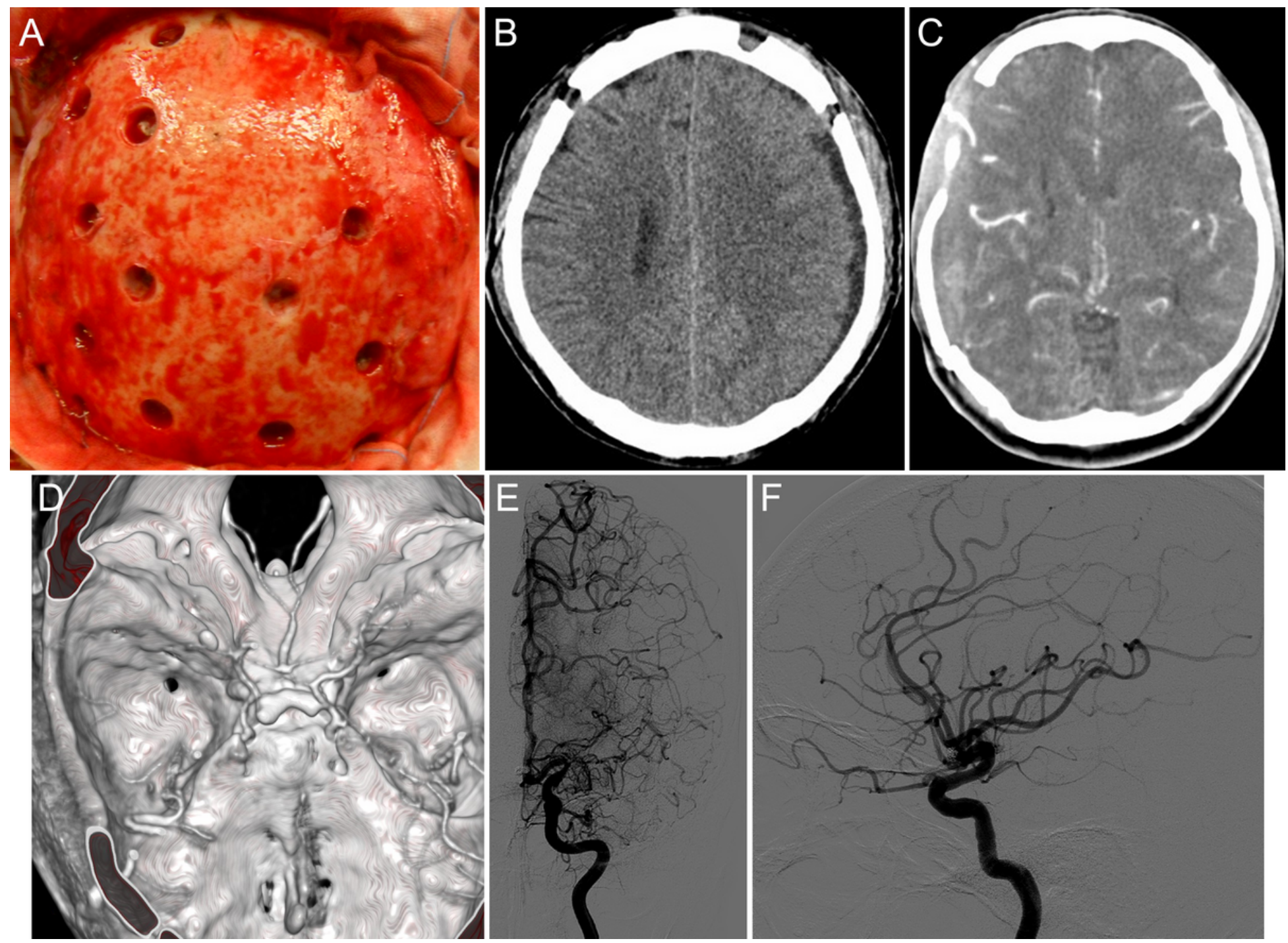

Figure 5

Treatment of MMD. (A, B) Multiple burr-hole operation (intraoperative photographic image and postoperative CT); (C, D) the connection of intracranial and extracranial vessels after bypass (CTA); (E, F) embolization of ACA aneurysm in MMD (frontal and lateral views, DSA). 